\title{
Stability Analysis of Discrete-Time Recurrent Neural Networks With Stochastic Delay
}

\author{
Yu Zhao, Huijun Gao, James Lam, Senior Member, IEEE, and Ke Chen
}

\begin{abstract}
This paper is concerned with the stability analysis of discrete-time recurrent neural networks (RNNs) with time delays as random variables drawn from some probability distribution. By introducing the variation probability of the time delay, a common delayed discrete-time RNN system is transformed into one with stochastic parameters. Improved conditions for the mean square stability of these systems are obtained by employing new Lyapunov functions and novel techniques are used to achieve delay dependence. The merit of the proposed conditions lies in its reduced conservatism, which is made possible by considering not only the range of the time delays, but also the variation probability distribution. A numerical example is provided to show the advantages of the proposed conditions.
\end{abstract}

Index Terms-Delay dependence, discrete-time recurrent neural networks (RNNs), mean square stability, stochastic time delay.

\section{INTRODUCTION}

B OTH theory and application of recurrent neural networks (RNNs) have enjoyed tremendous development in the last few decades. The existence of time delays, which are often encountered in the investigation of neural networks, has been recognized as a major cause of instability and performance deterioration of the system. The results in [1] and [16] have shown that the neuron property of delay has important influence on the dynamic properties of the neural network. In [6] and [7], results on stability of discrete-time systems with time-varying state delay have been obtained and delay-dependent output-feedback stabilization of discrete-time systems with time-varying state delay has been investigated. In [8] and [9], the global asymptotic stability criterion has been derived and delay-dependent state estimation method has been developed for delayed neural

Manuscript received February 20, 2008; revised December 06, 2008 and March 01, 2009; accepted March 24, 2009. First published July 14, 2009; current version published August 05, 2009. This work was supported in part by the National Natural Science Foundation of China under Grants 60825303 and 60834003, by 973 Project (2009CB320600), by the Heilongjiang Outstanding Youth Science Fund (JC200809), and by the Research Grants Council under code HKU 7031/06P.

Y. Zhao is with the Space Control and Inertial Technology Research Center, Harbin Institute of Technology, Harbin 150001, China and also with the University of Southern California, Los Angeles, CA 90089 USA

$\mathrm{H}$. Gao is with the Space Control and Inertial Technology Research Center, Harbin Institute of Technology, Harbin 150001, China (e-mail: hjgao@ece.ualberta.ca).

J. Lam is with the Department of Mechanical Engineering, University of Hong Kong, Hong Kong (e-mail: james.lam@hku.hk).

K. Chen is with the Department of Electrical and Computer Engineering, University of Alberta, Edmonton, AB T6G 2R3 Canada (e-mail: kchen1@ece.ualberta.ca).

Color versions of one or more of the figures in this paper are available online at http://ieeexplore.ieee.org.

Digital Object Identifier 10.1109/TNN.2009.2023379 networks. Both constant and time-varying time delays have attracted considerable attention throughout the world and many aspects of RNNs with time delays have been analyzed. A great number of results concerning the existence of an equilibrium point, global exponential stability, and the existence of periodic solutions have been reported in literature (see [2], [3], [19], [20], [24]-[28], [30], [31], [33], and the references therein).

Although RNNs are mostly studied in the continuous-time setting, they are often discretized for experimental or computational purposes. The dynamic characteristics of discrete-time neural networks have been extensively investigated, and many results have been obtained (see [6], [7], [10], [22], [29], [32], [34], [35], and references therein). In [10], the global robust stability has been analyzed for a general class of discrete-time interval neural networks which contain time-invariant uncertain parameters with their values being unknown but bounded in given compact sets. Some sufficient criteria have been derived in [29] and [34] in order to ensure the asymptotic stability of the equilibrium point for a discrete-time Cohen-Grossberg neural network model. In [35], the existence of periodic solutions has been proved for a nonautonomous discrete-time neural network by using the topological degree theory. In [15], the stability analysis problem of the exponential stability for a class of delayed discrete-time RNNs is investigated under a more general description on the activation functions, and less conservative stability criteria are obtained by using a unified linear matrix inequality (LMI) approach.

In [15], the following delayed discrete-time RNN system was considered:

$$
x(k+1)=C x(k)+A f(x(k))+B f(x(k-\tau(k)))+J(k)
$$

where $x(k) \in \mathbb{R}^{n}$ and $\tau(k)$ is a time-varying delay. Sufficient conditions for the stability of the system are derived in terms of the upper bound $\tau^{M}$ and lower bound $\tau^{m}$ of $\tau(k)$, that is, when $\tau^{m} \leq \tau(k) \leq \tau^{M}$. As most commonly established results, the proposed criteria in [15] employ only information of the variation range of the time delay. However, network tomography, the technique that is concerned with inference of delay distribution of networked systems, makes it possible that the probability distribution of time delay $\tau(k)$ in practical systems is known in advance (see [4], [5], [18], [21], and the references therein). This provides the theoretical basis for our assumption that delay probability distribution can be obtained in practical systems. When the probability distribution of $\tau(k)$ is available, it would be possible to utilize such information to reduce the conservatism of these criteria. In fact, as pointed out in [11] and [23], a more practical and precise description for a given discrete-time-delay RNNs is that there exists a small number $\varepsilon$ such 
that $\operatorname{Prob}\{\tau(k)>d\} \leq \varepsilon$ where $d$ is a constant. What needs to be investigated is finding an upper bound of $d$ for a given $\varepsilon$. There is certain probability of $\tau(k)$ to take values in $\left[\tau_{1}^{m}, \tau_{1}^{M}\right]$ and $\left[\tau_{2}^{m}, \tau_{2}^{M}\right]$, and $\tau(k)$ is a set of jumping values which may change its values from the intervals $\left[\tau_{1}^{m}, \tau_{1}^{M}\right]$ and $\left[\tau_{2}^{m}, \tau_{2}^{M}\right]$. It is obvious that this description is more general than the common time-delay systems considered in [15] and the variation probability of the time delay will affect the size of the allowable variation range. However, to the best of the authors' knowledge, little consideration has been given to applying the probability distribution of time-delay values to analyze the stability of discrete-time RNNs and this motivates this study.

In this paper, the stability of the solutions for discrete-time RNNs is investigated based on the information of both the variation range and the variation probability of the time delay. A new modeling method is presented to show the effects of distribution probability of the time delay, and a new Lyapunov function is employed for stability analysis. Based on Lyapunov method and stochastic analysis theory, some new criteria to guarantee the mean square stability of the system are derived using LMI approach. A numerical example is provided to illustrate the reduction of conservatism of the proposed criteria. According to the numerical example, when the variation probability of the time delay is given, the upper bound of the time delay that can be tolerated before instability occurs may be much larger than that when only the variation range of the time delay is known.

Notation: The notation used throughout this paper is fairly standard. The superscript " $T$ " stands for matrix transposition; $\mathbb{R}^{n}$ denotes the $n$-dimensional Euclidean space; and the notation $P>0(\geq 0)$ means that $P$ is real symmetric and positive definite (semidefinite). $I$ and 0 represent identity matrix and zero matrix, respectively. In symmetric block matrices or complex matrix expressions, we use an asterisk $*$ to represent a term that is induced by symmetry and $\operatorname{diag}\{\ldots\}$ stands for a block-diagonal matrix. Matrices, if their dimensions are not explicitly stated, are assumed to be compatible for algebraic operations. $E\{\cdot\}$ denotes the expectation and $E\{y \mid x\}$ denotes the expectation of $y$ conditional on $x$.

\section{PROBlem Description}

In the literature, the following delayed discrete-time RNN was considered:

$$
x(k+1)=C x(k)+A f(x(k))+B f(x(k-\tau(k)))+J
$$

where $x(k)=\left[x_{1}(k), x_{2}(k), \ldots, x_{n}(k)\right]^{T}$ is the neural state vector; $C=\operatorname{diag}\left\{c_{1}, c_{2}, \ldots, c_{n}\right\}$ with $\left|c_{i}\right|<1$ is the state feedback coefficient matrix; the $n \times n$ matrices $A=\left[a_{i j}\right]_{n \times n}$ and $B=\left[b_{i j}\right]_{n \times n}$ are the connection weight matrix and the delayed connection weight matrix, respectively; $f(x(k))$ denotes the neuron activation function, which satisfies

$$
f(x(k))=\left[f_{1}\left(x_{1}(k)\right), f_{2}\left(x_{2}(k)\right), \ldots, f_{n}\left(x_{n}(k)\right)\right]^{T}
$$

and the constant vector $J=\left[J_{1}, J_{2}, \ldots, J_{n}\right]^{T}$ is the exogenous input; and $\tau(k)$ denotes the time-varying delay. In practice, there is certain probability of time delay to take values in different intervals. That is, $\tau(k)$ takes values in $\left[\tau_{1}^{m}, \tau_{1}^{M}\right]$ and $\left[\tau_{2}^{m}, \tau_{2}^{M}\right]$ according to probability. Thus, $\tau(k)$ is a random variable which takes values in the intervals $\left[\tau_{1}^{m}, \tau_{1}^{M}\right]$ and $\left[\tau_{2}^{m}, \tau_{2}^{M}\right]$. Here, the probability distribution of $\tau(k)$ is assumed to be

$$
\begin{aligned}
& \operatorname{Prob}\left\{\tau_{1}^{m} \leq \tau(k) \leq \tau_{1}^{M}\right\}=\delta_{0} \\
& \operatorname{Prob}\left\{\tau_{2}^{m} \leq \tau(k) \leq \tau_{2}^{M}\right\}=1-\delta_{0} .
\end{aligned}
$$

To give a more precise and reasonable description of the system, we introduce a stochastic variable $\delta(k)$ by

$$
\delta(k)= \begin{cases}1, & \tau_{1}^{m} \leq \tau(k) \leq \tau_{1}^{M} \\ 0, & \tau_{2}^{m} \leq \tau(k) \leq \tau_{2}^{M} .\end{cases}
$$

Then, it can be derived that

$$
\begin{aligned}
\operatorname{Prob}\{\delta(k)=1\} & =\operatorname{Prob}\left\{\tau_{1}^{m} \leq \tau(k) \leq \tau_{1}^{M}\right\} \\
& =E\{\delta(k)\}=\delta_{0} \\
\operatorname{Prob}\{\delta(k)=0\} & =\operatorname{Prob}\left\{\tau_{2}^{m} \leq \tau(k) \leq \tau_{2}^{M}\right\} \\
& =1-E\{\delta(k)\}=1-\delta_{0}
\end{aligned}
$$

and

$$
\begin{aligned}
E\left\{\delta(k)-\delta_{0}\right\} & =0 \\
E\left\{\left(\delta(k)-\delta_{0}\right)^{2}\right\} & =\delta_{0}\left(1-\delta_{0}\right) .
\end{aligned}
$$

In terms of the definition of $\delta(k)$, the system in (1) can be rewritten as

$$
\begin{aligned}
x(k+1)=C x(k) & +A f(x(k))+\delta(k) B f\left(x\left(k-\tau_{1}(k)\right)\right) \\
& +(1-\delta(k)) B f\left(x\left(k-\tau_{2}(k)\right)\right)+J .
\end{aligned}
$$

The positive integers $\tau_{1}(k)$ and $\tau_{2}(k)$ denote the time-varying delay values satisfying

$$
\tau_{1}^{m} \leq \tau_{1}(k) \leq \tau_{1}^{M} \quad \tau_{2}^{m} \leq \tau_{2}(k) \leq \tau_{2}^{M}
$$

where $\tau_{1}^{m}, \tau_{1}^{M}, \tau_{2}^{m}$, and $\tau_{2}^{M}$ are known integers satisfying $0<$ $\tau_{1}^{m} \leq \tau_{1}^{M}<\tau_{2}^{m} \leq \tau_{2}^{M} \leq \tau$. Here, $\tau$ is a given integer which is used to describe the delay bound. Similar characterization of the delay has appeared in [20].

Assumption 1: The activation function $f_{i}(x) \quad(i=$ $1,2, \ldots, n)$ is continuous and bounded. Moreover, there exist constants $F_{i}^{+}$and $F_{i}^{-}(i=1,2, \ldots, n)$ such that

$$
F_{i}^{-} \leq \frac{f_{i}\left(\alpha_{1}\right)-f_{i}\left(\alpha_{2}\right)}{\alpha_{1}-\alpha_{2}} \leq F_{i}^{+}
$$

for all $\alpha_{1} \neq \alpha_{2}$.

Remark 1: The constants $F_{j}^{+}$and $F_{j}^{-}$in Assumption 1 are allowed to be positive, negative, or zero. Hence, the resulting activation functions could be nonmonotonic, and are more general than the usual sigmoid functions and the commonly used Lipschitz conditions. Note that with such a milder assumption, the analysis methods developed in [12] and [13] cannot be applied directly, and a new approach will have to be developed.

Remark 2: The delayed discrete-time RNN system in (1) has been considered in [15] where $\tau(k)$ is assumed to have the upper and lower bounds, that is, $\tau^{m} \leq \tau(k) \leq \tau^{M}$. In this paper, we assume certain probability distribution of the time delay. It is worth mentioning that when $\delta(k) \equiv 1$, which means $\tau(k)=\tau_{1}(k)$, (3) reduces to $x(k+1)=C x(k)+A f(x(k))+B f\left(x\left(k-\tau_{1}(k)\right)\right)+J$, 
which is exactly the system treated in [15]. In this sense, the model considered in this paper is more general than those in the literature.

Remark 3: A more general system model may include different delay in each component of the state vector. In this case, the system model can be described as follows:

$$
\begin{gathered}
x(k+1)=C x(k)+A f(x(k)) \\
+B\left[f_{1}\left(x_{1}\left(k-\tau_{1}(k)\right)\right), f_{2}\left(x_{2}\left(k-\tau_{2}(k)\right)\right),\right. \\
\left.\ldots, f_{n}\left(x_{n}\left(k-\tau_{n}(k)\right)\right)\right]^{T}+J .
\end{gathered}
$$

This model can further be transformed into

$$
x(k+1)=C x(k)+A f(x(k))+\sum_{i=1}^{n} B_{i} f\left(x\left(k-\tau_{i}(k)\right)\right)+J
$$

where $B_{i}$ denotes the matrix derived from the transformation. The method we employed in our paper is applicable to this general case, and the stability analysis can be conducted similarly. Our choice of the system gives a good lucidity of the exposition.

The following lemma will be used in subsequent derivations.

Lemma 1: Under Assumption 1, there exists an equilibrium point of the system in (3).

Proof: Since the activation functions are bounded, there exists a constant $M_{0}$ such that $|f(x)| \leq M_{0}$, $\forall x \in \mathbb{R}^{n}$. It follows from $\left|c_{i}\right|<1$ that $I-C$ is invertible. We denote $\xi(r)=\left\{x \in \mathbb{R}^{n}|| x \mid \leq r\right\}$ with $r=\left\|(I-C)^{-1}\right\|\left(\|A\| M_{0}+\|B\| M_{0}+|J|\right)$, and define the map $V: \xi \rightarrow \mathbb{R}^{n}$ by

$$
V(x)=(I-C)^{-1}(A f(x)+\delta B f(x)+(1-\delta) B f(x)+J)
$$

where $\delta$ is a stochastic parameter taking values in $[0,1]$. Obviously, $V$ is a continuous map, and it follows that

$$
\begin{aligned}
|V(x)| \leq & \left\|(I-C)^{-1}\right\|(\|A\||f(x)|+\delta\|B\||f(x)| \\
& +(1-\delta)\|B\||f(x)|+|J|) \\
\leq & \left\|(I-C)^{-1}\right\|\left(\|A\| M_{0}+\|B\| M_{0}+|J|\right) .
\end{aligned}
$$

Therefore, $V$ maps $\xi(r)$ into itself. By Brouwer's fixed point theorem, it can be inferred that there exists a fixed point $x^{*}$ of $V$, satisfying

$$
(I-C)^{-1}\left(A f\left(x^{*}\right)+B f\left(x^{*}\right)+J\right)=x^{*}
$$

which is equivalent to

$$
\begin{aligned}
x^{*} & =C x^{*}+A f\left(x^{*}\right)+B f\left(x^{*}\right)+J \\
& =C x^{*}+A f\left(x^{*}\right)+\delta B f\left(x^{*}\right)+(1-\delta) B f\left(x^{*}\right)+J .
\end{aligned}
$$

Lemma 1 means that there exists an equilibrium point of the system in (3). Then, we denote $x^{*}=\left[x_{1}^{*}, x_{2}^{*}, \ldots, x_{n}^{*}\right]^{T}$ as the equilibrium point of the system in (3). For convenience, we shift the equilibrium $x^{*}$ to the origin by letting

$$
\begin{aligned}
& y(k)=x(k)-x^{*} \\
& g(k)=f(x(k))-f\left(x^{*}\right)
\end{aligned}
$$

and the system in (3) can be transformed into

$$
\begin{aligned}
y(k+1)=C y(k)+A g(k) & +\delta(k) B g\left(k-\tau_{1}(k)\right) \\
& +(1-\delta(k)) B g\left(k-\tau_{2}(k)\right)
\end{aligned}
$$

where $y(k)$ is the state vector of the transformed system. Obviously, the stability of the system in (4) can ensure the stability of the system in (3).

Since the system in (4) involves a stochastic parameter, to investigate its stability, we need the following definition.

Definition 1: The system in (4) is said to be mean square stable (MSS) if there exists a scalar $c>0$ such that

$$
E\left\{\sum_{k=0}^{\infty}\|y(k)\|^{2}\right\} \leqslant c \sup _{-\tau \leq k \leq 0} E\left\{\|y(k)\|^{2}\right\}
$$

where the set of vectors $\{y(-\tau), y(-\tau+1), \ldots, y(0)\}$ gives the initial state of the system in (4).

Remark 4: Mean square stability is one type of stability criteria which applies to the systems with stochastic properties. A system that is MSS in the sense of Definition 1 will have the expectation of system state tend to zero as time tends to infinity for arbitrary initial state (that is, for arbitrary $y(i), i=$ $-\tau,-\tau+1, \ldots, 0)$. This stability criterion is of great practical value since many optimal control problems use the squared norm in their calculations, and this motivates us to investigate the condition for mean square stability in this paper.

\section{MAIN RESUlts}

In this section, we will derive some criteria which can guarantee (4) is MSS. First, we rewrite (4) as

$$
\begin{aligned}
y(k+1)= & C y(k)+A g(k) \\
& +\left(\delta(k)-\delta_{0}\right) B\left(g\left(k-\tau_{1}(k)\right)-g\left(k-\tau_{2}(k)\right)\right) \\
& +\delta_{0} B g\left(k-\tau_{1}(k)\right)+\left(1-\delta_{0}\right) B g\left(k-\tau_{2}(k)\right) .
\end{aligned}
$$

To facilitate our presentation, in the following, we denote

$$
\begin{aligned}
& F_{1}=\operatorname{diag}\left(F_{1}^{-} F_{1}^{+}, F_{2}^{-} F_{2}^{+}, \ldots, F_{n}^{-} F_{n}^{+}\right) \\
& F_{2}=\operatorname{diag}\left(\frac{F_{1}^{-}+F_{1}^{+}}{2}, \frac{F_{2}^{-}+F_{2}^{+}}{2}, \ldots, \frac{F_{n}^{-}+F_{n}^{+}}{2}\right) .
\end{aligned}
$$

To develop the mean square stability, we notice that the difference of the expectation of the constructed Lyapunov function $E\{V(k+1) \mid \chi(k)\}-E\{V(k) \mid \chi(k)\}$ can be upper bounded by a quadratic form involving a vector containing the terms in $V(k)$. In this way, the stability condition can be inferred by the negative definiteness of the matrix associated with the quadratic form which, by applying Schur complement, can be converted into an LMI of some matrix variables. Since LMIs can be numerically solved conveniently, this transformation of the stability condition to an LMI form facilitates the check of stability. We are in a position to give the main result.

Theorem 1: Under Assumption 1, the system in (4) is MSS if there exist matrices $P>0, Q_{1}>0, Q_{2}>0, Z_{1}>0$, and 
$Z_{2}>0$, diagonal matrices $D>0, H>0$, and $L>0$, and matrices $S$ and $T$ such that the following LMI holds:

$$
W=\left[\begin{array}{cc}
W_{1}+\Xi+\Xi^{T} & W_{2} \\
* & -W_{3}
\end{array}\right]<0
$$

where

$$
\begin{aligned}
& W_{1}=\left[\begin{array}{ccc}
\Pi_{1}-F_{1} D & 0 & 0 \\
* & -Q_{1}-F_{1} H & 0 \\
* & * & -Q_{2}-F_{1} L \\
* & * & * \\
* & * & * \\
* & * & *
\end{array}\right. \\
& \Pi_{2}+F_{2} D \quad \Pi_{3} \\
& 0 \quad F_{2} H \\
& \begin{array}{lll}
0 & 0 & F_{2} L
\end{array} \\
& \Pi_{5}-D \quad \Pi_{6} \quad \Pi_{7} \\
& \text { * } \quad \Pi_{8}-H \quad 0 \\
& \Xi=[S+T,-S,-T, 0,0,0] \\
& W_{2}=\left[\sqrt{\tau_{1}^{M}} S, \sqrt{\tau_{2}^{M}} T\right] \\
& W_{3}=\operatorname{diag}\left(Z_{1}, Z_{2}\right)
\end{aligned}
$$

and

$$
\begin{aligned}
\Pi_{1}= & C^{T} P C-P+\left(\tau_{1}^{M}-\tau_{1}^{m}+1\right) Q_{1}+\left(\tau_{2}^{M}-\tau_{2}^{m}+1\right) Q_{2} \\
& +(C-I)^{T}\left(\tau_{1}^{M} Z_{1}+\tau_{2}^{M} Z_{2}\right)(C-I) \\
\Pi_{2}= & C^{T} P A+(C-I)^{T}\left(\tau_{1}^{M} Z_{1}+\tau_{2}^{M} Z_{2}\right) A \\
\Pi_{3}= & \delta_{0}\left(C^{T} P+(C-I)^{T}\left(\tau_{1}^{M} Z_{1}+\tau_{2}^{M} Z_{2}\right)\right) B \\
\Pi_{4}= & \left(1-\delta_{0}\right)\left(C^{T} P+(C-I)^{T}\left(\tau_{1}^{M} Z_{1}+\tau_{2}^{M} Z_{2}\right)\right) B \\
\Pi_{5}= & A^{T}\left(P+\tau_{1}^{M} Z_{1}+\tau_{2}^{M} Z_{2}\right) A \\
\Pi_{6}= & \delta_{0} A^{T}\left(P+\tau_{1}^{M} Z_{1}+\tau_{2}^{M} Z_{2}\right) B \\
\Pi_{7}= & \left(1-\delta_{0}\right) A^{T}\left(P+\tau_{1}^{M} Z_{1}+\tau_{2}^{M} Z_{2}\right) B \\
\Pi_{8}= & \delta_{0} B^{T}\left(P+\tau_{1}^{M} Z_{1}+\tau_{2}^{M} Z_{2}\right) B \\
\Pi_{9}= & \left(1-\delta_{0}\right) B^{T}\left(P+\tau_{1}^{M} Z_{1}+\tau_{2}^{M} Z_{2}\right) B .
\end{aligned}
$$

Proof: Construct a Lyapunov function as

$$
V(k)=V_{1}(k)+V_{2}(k)+V_{3}(k)+V_{4}(k)
$$

where

$$
\begin{aligned}
V_{1}(k)= & y^{T}(k) P y(k) \\
V_{2}(k)= & \sum_{i=k-\tau_{1}(k)}^{k-1} y^{T}(i) Q_{1} y(i) \\
& +\sum_{i=-\tau_{1}^{M}+1}^{-\tau_{1}^{m}} \sum_{j=k+i}^{k-1} y^{T}(j) Q_{1} y(j) \\
V_{3}(k)= & \sum_{i=k-\tau_{2}(k)}^{k-1} y^{T}(i) Q_{2} y(i) \\
& +\sum_{i=-\tau_{2}^{M}+1}^{-\tau_{2}^{m}} \sum_{j=k+i}^{k-1} y^{T}(j) Q_{2} y(j) \\
V_{4}(k)= & \sum_{i=-\tau_{1}^{M}}^{-1} \sum_{l=k+i}^{k-1} \eta^{T}(l) Z_{1} \eta(l)
\end{aligned}
$$

$$
\begin{gathered}
+\sum_{\substack{i=-\tau_{2}^{M} \\
l=k+i}}^{-1} \sum_{l=k}^{T-1} \eta^{T}(l) Z_{2} \eta(l) \\
\eta(k)=y(k+1)-y(k) .
\end{gathered}
$$

Here, $V_{1}(k)$ is the common term in the Lyapunov function. The first parts of $V_{2}(k)$ and $V_{3}(k)$ are common terms in the Lyapunov function constructed for delayed systems. The second parts of $V_{2}(k)$ and $V_{3}(k)$ are introduced for the systems with time-varying delay. $V_{4}(k)$ is constructed so that the derived condition is delay dependent. Denoting $\chi(k)=\{y(k), y(k-$ $1), \ldots, y(k-\tau(k))\}$, it follows:

$$
\begin{aligned}
E\left\{V_{i}(k+1) \mid \chi(k)\right\}-E\left\{V_{i}(k) \mid \chi(k)\right\} & \\
& =E\left\{V_{i}(k+1) \mid \chi(k)\right\}-V_{i}(k) .
\end{aligned}
$$

Therefore, based on (2) and (5), it can be shown that

$$
\begin{aligned}
E\left\{V_{1}\right. & (k+1) \mid \chi(k)\}-E\left\{V_{1}(k) \mid \chi(k)\right\} \\
= & E\{(C y(k)+A g(k) \\
& +\left(\delta(k)-\delta_{0}\right) B\left(g\left(k-\tau_{1}(k)\right)-g\left(k-\tau_{2}(k)\right)\right) \\
& \left.+\delta_{0} B g\left(k-\tau_{1}(k)\right)+\left(1-\delta_{0}\right) B g\left(k-\tau_{2}(k)\right)\right)^{T} \\
& \times P(C y(k)+A g(k) \\
& +\left(\delta(k)-\delta_{0}\right) B\left(g\left(k-\tau_{1}(k)\right)-g\left(k-\tau_{2}(k)\right)\right) \\
& +\delta_{0} B g\left(k-\tau_{1}(k)\right) \\
& \left.\left.+\left(1-\delta_{0}\right) B g\left(k-\tau_{2}(k)\right)\right)-y^{T}(k) P y(k)\right\} \\
= & y^{T}(k)\left(C^{T} P C-P\right) y(k)+2 y^{T}(k) C^{T} P A g(k) \\
& +2 \delta_{0} y^{T}(k) C^{T} P B g\left(k-\tau_{1}(k)\right) \\
& +2\left(1-\delta_{0}\right) y^{T}(k) C^{T} P B g\left(k-\tau_{2}(k)\right) \\
& +g^{T}(k) A^{T} P A g(k)+2 \delta_{0} g^{T}(k) A^{T} P B g\left(k-\tau_{1}(k)\right) \\
& +2\left(1-\delta_{0}\right) g^{T}(k) A^{T} P B g\left(k-\tau_{2}(k)\right) \\
& +\delta_{0}\left(1-\delta_{0}\right)\left(g\left(k-\tau_{1}(k)\right)-g\left(k-\tau_{2}(k)\right)\right)^{T} B^{T} \\
& \times P B\left(g\left(k-\tau_{1}(k)\right)-g\left(k-\tau_{2}(k)\right)\right) \\
& +\delta_{0}^{2} g^{T}\left(k-\tau_{1}(k)\right) B^{T} P B g\left(k-\tau_{1}(k)\right) \\
& +2 \delta_{0}\left(1-\delta_{0}\right) g^{T}\left(k-\tau_{1}(k)\right) B^{T} P B g\left(k-\tau_{2}(k)\right) \\
& +\left(1-\delta_{0}\right)^{2} g^{T}\left(k-\tau_{2}(k)\right) B^{T} P B g\left(k-\tau_{2}(k)\right)
\end{aligned}
$$

and

$$
\begin{aligned}
E\{ & \left.V_{2}(k+1) \mid \chi(k)\right\}-E\left\{V_{2}(k) \mid \chi(k)\right\} \\
= & \sum_{i=k+1-\tau_{1}(k+1)}^{k} y^{T}(i) Q_{1} y(i) \\
& +\sum_{i=-\tau_{1}^{M}+1}^{-\tau_{1}^{m}} \sum_{j=k+1+i}^{k} y^{T}(j) Q_{1} y(j) \\
& -\sum_{i=k-\tau_{1}(k)}^{k-1} y^{T}(i) Q_{1} y(i) \\
& -\sum_{i=-\tau_{1}^{M}+1}^{-\tau_{1}^{m}} \sum_{j=k+i}^{k-1} y^{T}(j) Q_{1} y(j) \\
= & \sum_{i=k+1-\tau_{1}^{m}}^{k} y^{T}(i) Q_{1} y(i)
\end{aligned}
$$




$$
\begin{aligned}
& +\sum_{i=k+1-\tau_{1}^{m}}^{k-1} y^{T}(i) Q_{1} y(i)+y^{T}(k) Q_{1} y(k) \\
& -\sum_{i=k-\tau_{1}(k)+1}^{k-1} y^{T}(i) Q_{1} y(i) \\
& -y^{T}\left(k-\tau_{1}(k)\right) Q_{1} y\left(k-\tau_{1}(k)\right) \\
& +\sum_{i=-\tau_{1}^{M}+1}^{-\tau_{1}^{m}}\left(y^{T}(k) Q_{1} y(k)-y^{T}(k+i) Q_{1} y(k+i)\right) \\
& \leq \sum_{i=k+1-\tau_{1}(k+1)}^{k-\tau_{1}^{m}} y^{T}(i) Q_{1} y(i)+\left(\tau_{1}^{M}-\tau_{1}^{m}+1\right) \\
& \times y^{T}(k) Q_{1} y(k)-y^{T}\left(k-\tau_{1}(k)\right) Q_{1} y\left(k-\tau_{1}(k)\right) \\
& -\sum_{i=-\tau_{1}^{M}+1}^{-\tau_{1}^{m}} y^{T}(k+i) Q_{1} y(k+i) \\
& \leq \\
& \quad\left(\tau_{1}^{M}-\tau_{1}^{m}+1\right) y^{T}(k) Q_{1} y(k) \\
& -y^{T}\left(k-\tau_{1}(k)\right) Q_{1} y\left(k-\tau_{1}(k)\right)
\end{aligned}
$$

and

$$
\begin{gathered}
E\left\{V_{3}(k+1) \mid \chi(k)\right\}-E\left\{V_{3}(k) \mid \chi(k)\right\} \\
\leq\left(\tau_{2}^{M}-\tau_{2}^{m}+1\right) y^{T}(k) Q_{2} y(k) \\
\quad-y^{T}\left(k-\tau_{2}(k)\right) Q_{2} y\left(k-\tau_{2}(k)\right)
\end{gathered}
$$

and

$$
\begin{aligned}
E\left\{V_{4}(k+1) \mid \chi(k)\right\}-E\left\{V_{4}(k) \mid \chi(k)\right\} & \left\{\sum_{i=-\tau_{1}^{M}}^{-1}\left[\eta^{T}(k) Z_{1} \eta(k)-\eta^{T}(k+i) Z_{1} \eta(k+i)\right]\right. \\
= & \left.\quad+\sum_{i=-\tau_{2}^{M}}^{-1}\left[\eta^{T}(k) Z_{2} \eta(k)-\eta^{T}(k+i) Z_{2} \eta(k+i)\right]\right\} \\
& \\
& E\left\{\tau_{1}^{M} \eta^{T}(k) Z_{1} \eta(k)\right\}-\sum_{i=k-\tau_{1}^{M}}^{k-1} \eta^{T}(i) Z_{1} \eta(i) \\
& +E\left\{\tau_{2}^{M} \eta^{T}(k) Z_{2} \eta(k)\right\}-\sum_{i=k-\tau_{2}^{M}}^{k-1} \eta^{T}(i) Z_{2} \eta(i) \\
= & y^{T}(k)(C-I)^{T}\left(\tau_{1}^{M} Z_{1}+\tau_{2}^{M} Z_{2}\right)(C-I) y(k) \\
& +2 y^{T}(k)(C-I)^{T}\left(\tau_{1}^{M} Z_{1}+\tau_{2}^{M} Z_{2}\right) A g(k) \\
& +2 \delta_{0} y^{T}(k)(C-I)^{T}\left(\tau_{1}^{M} Z_{1}+\tau_{2}^{M} Z_{2}\right) B g\left(k-\tau_{1}(k)\right) \\
& +2\left(1-\delta_{0}\right) y^{T}(k)(C-I)^{T} \\
& \times\left(\tau_{1}^{M} Z_{1}+\tau_{2}^{M} Z_{2}\right) B g\left(k-\tau_{2}(k)\right) \\
& +g^{T}(k) A^{T}\left(\tau_{1}^{M} Z_{1}+\tau_{2}^{M} Z_{2}\right) A g(k) \\
& +2 \delta_{0} g^{T}(k) A^{T}\left(\tau_{1}^{M} Z_{1}+\tau_{2}^{M} Z_{2}\right) B g\left(k-\tau_{1}(k)\right) \\
& +2\left(1-\delta_{0}\right) g^{T}(k) A^{T}\left(\tau_{1}^{M} Z_{1}+\tau_{2}^{M} Z_{2}\right) B g\left(k-\tau_{2}(k)\right) \\
& +\delta_{0} g^{T}\left(k-\tau_{1}(k)\right) B^{T}\left(\tau_{1}^{M} Z_{1}+\tau_{2}^{M} Z_{2}\right) B g\left(k-\tau_{1}(k)\right) \\
& +\left(1-\delta_{0}\right) g^{T}\left(k-\tau_{2}(k)\right) B^{T} \\
& \times\left(\tau_{1}^{M} Z_{1}+\tau_{2}^{M} Z_{2}\right) B g\left(k-\tau_{2}(k)\right) \\
& -\sum_{i=k-1}^{k-1} \eta^{T}(i) Z_{1} \eta(i)-\sum_{1}^{k-1} \eta^{T}(i) Z_{2} \eta(i) . \\
& \sum_{i=k-\tau_{1}}
\end{aligned}
$$

Let

$$
\begin{array}{r}
\alpha(k)=\left[y^{T}(k), y^{T}\left(k-\tau_{1}(k)\right), y^{T}\left(k-\tau_{2}(k)\right),\right. \\
\left.g^{T}(k), g^{T}\left(k-\tau_{1}(k)\right), g^{T}\left(k-\tau_{2}(k)\right)\right]^{T} \\
\Pi=\left[\begin{array}{cccccc}
\Pi_{1} & 0 & 0 & \Pi_{2} & \Pi_{3} & \Pi_{4} \\
* & -Q_{1} & 0 & 0 & 0 & 0 \\
* & * & -Q_{2} & 0 & 0 & 0 \\
* & * & * & \Pi_{5} & \Pi_{6} & \Pi_{7} \\
* & * & * & * & \Pi_{8} & 0 \\
* & * & * & * & * & \Pi_{9}
\end{array}\right] .
\end{array}
$$

Then, it follows from (8)-(11) that

$E\{V(k+1) \mid \chi(k)\}-E\{V(k) \mid \chi(k)\}$

$$
\begin{aligned}
\leq & y^{T}(k)\left(C^{T} P C-P+\left(\tau_{1}^{M}-\tau_{1}^{m}+1\right) Q_{1}\right. \\
& +\left(\tau_{2}^{M}-\tau_{2}^{m}+1\right) Q_{2}+(C-I)^{T} \\
& \left.\times\left(\tau_{1}^{M} Z_{1}+\tau_{2}^{M} Z_{2}\right)(C-I)\right) y(k) \\
& -y^{T}\left(k-\tau_{1}(k)\right) Q_{1} y\left(k-\tau_{1}(k)\right) \\
& -y^{T}\left(k-\tau_{2}(k)\right) Q_{2} y\left(k-\tau_{2}(k)\right) \\
& +2 y^{T}(k)\left(C^{T} P+(C-I)^{T}\left(\tau_{1}^{M} Z_{1}+\tau_{2}^{M} Z_{2}\right)\right) A g(k) \\
& +2 \delta_{0} y^{T}(k)\left(C^{T} P+(C-I)^{T}\left(\tau_{1}^{M} Z_{1}+\tau_{2}^{M} Z_{2}\right)\right) \\
& \times B g\left(k-\tau_{1}(k)\right)+2\left(1-\delta_{0}\right) y^{T}(k) \\
& \times\left(C^{T} P+(C-I)^{T}\left(\tau_{1}^{M} Z_{1}+\tau_{2}^{M} Z_{2}\right)\right) B g\left(k-\tau_{2}(k)\right) \\
& +g^{T}(k) A^{T}\left(P+\tau_{1}^{M} Z_{1}+\tau_{2}^{M} Z_{2}\right) A g(k) \\
& +2 \delta_{0} g^{T}(k) A^{T}\left(P+\tau_{1}^{M} Z_{1}+\tau_{2}^{M} Z_{2}\right) B g\left(k-\tau_{1}(k)\right) \\
& +2\left(1-\delta_{0}\right) g^{T}(k) A^{T}\left(P+\tau_{1}^{M} Z_{1}+\tau_{2}^{M} Z_{2}\right) B g\left(k-\tau_{2}(k)\right) \\
& \times+\delta_{0} g^{T}\left(k-\tau_{1}(k)\right) B^{T}\left(P+\tau_{1}^{M} Z_{1}+\tau_{2}^{M} Z_{2}\right) \\
& \times B g\left(k-\tau_{1}(k)\right)+\left(1-\delta_{0}\right) g^{T}\left(k-\tau_{2}(k)\right) B^{T} \\
& \times\left(P+\tau_{1}^{M} Z_{1}+\tau_{2}^{M} Z_{2}\right) B g\left(k-\tau_{2}(k)\right) \\
& -\sum_{i=k-\tau_{1}^{M}}^{k-1} \eta^{T}(i) Z_{1} \eta(i)-\sum_{i=k-\tau_{2}^{M}}^{k-1} \eta^{T}(i) Z_{2} \eta(i) \\
\leq & \alpha^{T}(k) \Pi \alpha(k)-\sum_{i=k-\tau_{1}(k)}^{k-1} \eta^{T}(i) Z_{1} \eta(i) \\
& -\sum_{i=k-\tau_{2}(k)}^{k-1} \eta^{T}(i) Z_{2} \eta(i) . \\
&
\end{aligned}
$$

By introducing free-weighting matrices which are used to eliminate the undesirable terms in (12), it can be easily seen that (12) is equivalent to

$$
\begin{aligned}
& E\{V(k+1) \mid \chi(k)\}-E\{V(k) \mid \chi(k)\} \\
& \leq \alpha^{T}(k) \Pi \alpha(k)-\sum_{i=k-\tau_{1}(k)}^{k-1} \eta^{T}(i) Z_{1} \eta(i) \\
& \quad-\sum_{i=k-\tau_{2}(k)}^{k-1} \eta^{T}(i) Z_{2} \eta(i) \\
& \quad+2 \alpha^{T}(k) S\left(y(k)-y\left(k-\tau_{1}(k)\right)+\sum_{i=k-\tau_{1}(k)}^{k-1} \eta(i)\right) \\
& \quad+2 \alpha^{T}(k) T\left(y(k)-y\left(k-\tau_{2}(k)\right)+\sum_{i=k-\tau_{2}(k)}^{k-1} \eta(i)\right) .
\end{aligned}
$$


It is obvious that for $i$ satisfying $k-\tau_{1}(k) \leq i \leq k-1$

$$
0 \leq \alpha^{T}(k) S Z_{1}^{-1} S^{T} \alpha(k)+\eta^{T}(i) Z_{1} \eta(i)-2 \alpha^{T}(k) S \eta(i)
$$

we use certain weight matrix as coefficients for $\alpha(k)$ and $\eta(i)$ to make the term including $\alpha(k)$ and $\eta(i)$ agree in dimension, and also to eliminate certain terms in the future derivation. Adding $\tau_{1}(k)$ inequalities together, we obtain

$0 \leq \tau_{1}(k) \alpha^{T}(k) S Z_{1}^{-1} S^{T} \alpha(k)$

$$
+\sum_{i=k-\tau_{1}(k)}^{k-1} \eta^{T}(i) Z_{1} \eta(i)-2 \alpha^{T}(k) S \sum_{i=k-\tau_{1}(k)}^{k-1} \eta(i) .
$$

Since $\tau_{1}(k) \leq \tau_{1}^{M}$, we further obtain

$$
\begin{aligned}
0 \leq \tau_{1}^{M} \alpha^{T}(k) S Z_{1}^{-1} S^{T} \alpha(k) & \\
& +\sum_{i=k-\tau_{1}(k)}^{k-1} \eta^{T}(i) Z_{1} \eta(i)-2 \alpha^{T}(k) S \sum_{i=k-\tau_{1}(k)}^{k-1} \eta(i)
\end{aligned}
$$

which is equivalent to

$$
\begin{aligned}
& 2 \alpha^{T}(k) S \sum_{i=k-\tau_{1}(k)}^{k-1} \eta(i) \\
& \quad \leq \tau_{1}^{M} \alpha^{T}(k) S Z_{1}^{-1} S^{T} \alpha(k)+\sum_{i=k-\tau_{1}(k)}^{k-1} \eta^{T}(i) Z_{1} \eta(i)
\end{aligned}
$$

and similarly, we can obtain

$$
\begin{aligned}
& 2 \alpha^{T}(k) T \sum_{i=k-\tau_{2}(k)}^{k-1} \eta(i) \\
& \quad \leq \tau_{2}^{M} \alpha^{T}(k) T Z_{2}^{-1} T^{T} \alpha(k)+\sum_{i=k-\tau_{2}(k)}^{k-1} \eta^{T}(i) Z_{2} \eta(i) .
\end{aligned}
$$

Substituting (14) and (15) into (13) and taking expectation on both sides of (13), we obtain

$$
\begin{aligned}
& E\{V(k+1) \mid \chi(k)\}-E\{V(k) \mid \chi(k)\} \\
& \quad \leq \alpha^{T}(k)\left(\Pi+\Xi+\Xi^{T}+\tau_{1}^{M} S Z_{1}^{-1} S^{T}+\tau_{2}^{M} T Z_{2}^{-1} T^{T}\right) \alpha(k) .
\end{aligned}
$$

From Assumption 1, we have

$$
\begin{aligned}
& \left(g_{j}(k)-F_{j}^{-} y_{j}(k)\right)\left(g_{j}(k)-F_{j}^{+} y_{j}(k)\right) \leq 0 \\
& \left(g_{j}\left(k-\tau_{1}(k)\right)-F_{j}^{-} y_{j}\left(k-\tau_{1}(k)\right)\right) \\
& \quad \times\left(g_{j}\left(k-\tau_{1}(k)\right)-F_{j}^{+} y_{j}\left(k-\tau_{1}(k)\right)\right) \leq 0 \\
& \left(g_{j}\left(k-\tau_{2}(k)\right)-F_{j}^{-} y_{j}\left(k-\tau_{2}(k)\right)\right) \\
& \quad \times\left(g_{j}\left(k-\tau_{2}(k)\right)-F_{j}^{+} y_{j}\left(k-\tau_{2}(k)\right)\right) \leq 0
\end{aligned}
$$

which are equivalent to

$$
\begin{aligned}
& {\left[\begin{array}{l}
y(k) \\
g(k)
\end{array}\right]^{T}\left[\begin{array}{cc}
F_{j}^{-} F_{j}^{+} e_{j} e_{j}^{T} & -\frac{F_{j}^{+}+F_{j}^{-}}{2} e_{j} e_{j}^{T} \\
-\frac{F_{j}^{+}+F_{j}^{-}}{2} e_{j} e_{j}^{T} & e_{j} e_{j}^{T}
\end{array}\right]} \\
& \times\left[\begin{array}{l}
y(k) \\
g(k)
\end{array}\right] \leq 0
\end{aligned}
$$

$$
\begin{aligned}
& {\left[\begin{array}{c}
y\left(k-\tau_{1}(k)\right) \\
g\left(k-\tau_{1}(k)\right)
\end{array}\right]^{T}\left[\begin{array}{cc}
F_{j}^{-} F_{j}^{+} e_{j} e_{j}^{T} & -\frac{F_{j}^{+}+F_{j}^{-}}{2} e_{j} e_{j}^{T} \\
-\frac{F_{j}^{+}+F_{j}^{-}}{2} e_{j} e_{j}^{T} & e_{j} e_{j}^{T}
\end{array}\right]} \\
& \times\left[\begin{array}{l}
y\left(k-\tau_{1}(k)\right) \\
g\left(k-\tau_{1}(k)\right)
\end{array}\right] \leq 0 \\
& {\left[\begin{array}{l}
y\left(k-\tau_{2}(k)\right) \\
g\left(k-\tau_{2}(k)\right)
\end{array}\right]^{T}\left[\begin{array}{c}
F_{j}^{-} F_{j}^{+} e_{j} e_{j}^{T} \\
-\frac{F_{j}^{+}+F_{j}^{-}}{2} e_{i} e_{i}^{T}
\end{array}\right.} \\
& \quad \times\left[\begin{array}{l}
y\left(k-\tau_{2}(k)\right) \\
g\left(k-\tau_{2}(k)\right)
\end{array}\right] \leq 0
\end{aligned}
$$

where $j=1,2, \ldots, n$ and $e_{r}$ denotes the unit column vector having one element on its $r$ th row and zeros elsewhere. Denoting positive diagonal matrices $D=\operatorname{diag}\left\{d_{1}, d_{2}, \ldots, d_{n}\right\}$, $H=\operatorname{diag}\left\{h_{1}, h_{2}, \ldots, h_{n}\right\}$, and $L=\operatorname{diag}\left\{l_{1}, l_{2}, \ldots, l_{n}\right\}$, we have the equation shown at the bottom of the next page.

Applying Schur complement to (6), we obtain that there exists a scalar $\lambda>0$ such that

$$
W_{1}+\Xi+\Xi^{T}+\tau_{1}^{M} S Z_{1}^{-1} S^{T}+\tau_{2}^{M} T Z_{2}^{-1} T^{T}<-\lambda I .
$$

Therefore

$$
\begin{aligned}
E\{V(k+1)\}-E\{V(k)\} & <-\lambda E\left\{\|\alpha(k)\|^{2}\right\} \\
& \leq-\lambda E\left\{\|y(k)\|^{2}\right\} .
\end{aligned}
$$

From (16), it can be easily computed that

$$
E\left\{\sum_{k=0}^{\infty}\|y(k)\|^{2}\right\} \leq \frac{1}{\lambda} E\{V(0)\} .
$$

From the definition of $V(k)$, it can be shown that a scalar $c>0$ can be found such that

$$
E\{V(0)\} \leq \lambda c \sup _{-\tau \leq k \leq 0} E\left\{\|y(k)\|^{2}\right\} .
$$

Combining (17) and (18), we finally conclude that

$$
E\left\{\sum_{k=0}^{\infty}\|y(k)\|^{2}\right\} \leq c \sup _{-\tau \leq k \leq 0} E\left\{\|y(k)\|^{2}\right\}
$$

and the proof is completed.

Remark 5: To facilitate understanding, we summarize the respective roles of the matrices involved in matrix $W$ in Theorem 1. Matrix $W$ involves matrices $P, Q_{1}, Q_{2}, Z_{1}, Z_{2}, F_{1}, F_{2}, D$, $H, L, S$, and $T$. Matrices $P, Q_{1}, Q_{2}, Z_{1}$, and $Z_{2}$ are used to construct the Lyapunov function. Matrices $F_{1}$ and $F_{2}$ are used to characterize the bounds of the condition in Assumption 1, and they reflect the properties of the activation functions. Matrices $D, H$, and $L$ are the weights for the group of inequalities which are derived from Assumption 1. They are chosen so that the inequalities derived from Assumption 1 can be combined with the inequalities derived from the Lyapunov function. $S$ and $T$ are free-weighting matrices. They are introduced to eliminate the terms $\sum_{i=k-\tau_{1}(k)}^{k-1} \eta^{T}(i) Z_{1} \eta(i)$ and $\sum_{i=k-\tau_{2}(k)}^{k-1} \eta^{T}(i) Z_{2} \eta(i)$ in (11). The introduction of the free-weighting matrices leads to a less conservative result. 
As pointed out in Section I, when $\delta(k) \equiv 1$, that is, there is only one delay interval with $\tau(k)=\tau_{1}(k)$, system (4) is equivalent to

$$
y(k+1)=C y(k)+A g(k)+B g(k-\tau(k)) .
$$

In this case, $\tau(k)$ takes values in the interval $\left[\tau^{m}, \tau^{M}\right]$. Based on Theorem 1, the following corollary is obtained.

Corollary 1: The system in (19) is asymptotically stable, if there exist matrices $P>0, Q>0$, and $Z>0$, diagonal matrices $D>0$ and $H>0$, and matrix $S$ such that the following LMI holds:

$$
W=\left[\begin{array}{cc}
W_{1}+\Xi+\Xi^{T} & \sqrt{\tau^{M}} S \\
* & -Z
\end{array}\right]<0
$$

where

$$
\begin{aligned}
W_{1} & =\left[\begin{array}{cccc}
\Pi_{1}-F_{1} D & 0 & \Pi_{2}+F_{2} D & \Pi_{3} \\
* & -Q-F_{1} H & 0 & F_{2} H \\
* & * & \Pi_{4}-D & \Pi_{5} \\
* & * & * & \Pi_{6}-H
\end{array}\right] \\
\Xi & =[S,-S, 0,0]
\end{aligned}
$$

and

$$
\begin{aligned}
& \Pi_{1}=C^{T} P C-P+\left(\tau^{M}-\tau^{m}+1\right) Q+\tau^{M}(C-I)^{T} Z(C-I) \\
& \Pi_{2}=C^{T} P A+\tau^{M}(C-I)^{T} Z A \\
& \Pi_{3}=C^{T} P B+\tau^{M}(C-I)^{T} Z B \\
& \Pi_{4}=A^{T}\left(P+\tau^{M} Z\right) A \\
& \Pi_{5}=A^{T}\left(P+\tau^{M} Z\right) B \\
& \Pi_{6}=B^{T}\left(P+\tau^{M} Z\right) B .
\end{aligned}
$$

Remark 6: Our proposed criteria are less conservative than previous results for the reason that the theorems in the literature can be obtained by selecting special structures for the matrix variables in Corollary 1. Let us select the following structures in Corollary 1:

$$
S=0 \quad Z=\epsilon I
$$

with $\epsilon$ being a sufficiently small positive constant. Then, condition $(20)$ reduces to

$$
\begin{aligned}
W & =\left[\begin{array}{cccc}
\Pi_{1}-F_{1} D & 0 & C^{T} P A+F_{2} D & C^{T} P B \\
* & -Q-F_{1} H & 0 & F_{2} H \\
* & * & A^{T} P A-D & A^{T} P B \\
* & * & * & B^{T} P B-H
\end{array}\right] \\
& <0
\end{aligned}
$$

where

$$
\Pi_{1}=C^{T} P C-P+\left(\tau^{M}-\tau^{m}+1\right) Q
$$

which is equivalent to [15, Th. 1]. Hence, Corollary 1 is less conservative than [15, Th. 1].

Remark 7: In our main result, the stability analysis problems are dealt with for a general class of discrete-time neural networks with time-varying delays. An LMI-based sufficient condition is derived for the mean square stability of the neural networks under consideration. The mean square stability can be checked by the solvability of a set of LMIs, which can be done by resorting to Matlab LMI toolbox.

Remark 8: It is noticed that in Theorem 1 both the variation range of the time delay and the probability of the time delay are considered while in previous research (see [14], [15], and [17]) only the variation range of the time delay was investigated. Therefore, Theorem 1 is less conservative than the criteria derived in the literature.

$$
\begin{aligned}
& E\{V(k+1)\}-E\{V(k)\} \\
& \leq E\left\{\alpha^{T}(k)\left(\Pi+\Xi+\Xi^{T}+\tau_{1}^{M} S Z_{1}^{-1} S^{T}+\tau_{2}^{M} T Z_{2}^{-1} T^{T}\right) \alpha(k)-\sum_{i=1}^{n} d_{i}\left[\begin{array}{l}
y(k) \\
g(k)
\end{array}\right]^{T}\left[\begin{array}{cc}
F_{j}^{-} F_{j}^{+} e_{j} e_{j}^{T} & -\frac{F_{j}^{+}+F_{j}^{-}}{2} e_{j} e_{j}^{T} \\
-\frac{F_{j}^{+}+F_{j}^{-}}{2} e_{j} e_{j}^{T} & e_{j} e_{j}^{T}
\end{array}\right]\right. \\
& \times\left[\begin{array}{l}
y(k) \\
g(k)
\end{array}\right]-\sum_{i=1}^{n} h_{i}\left[\begin{array}{l}
y\left(k-\tau_{1}(k)\right) \\
g\left(k-\tau_{1}(k)\right)
\end{array}\right]^{T}\left[\begin{array}{cc}
F_{j}^{-} F_{j}^{+} e_{j} e_{j}^{T} & -\frac{F_{j}^{+}+F_{j}^{-}}{2} e_{j} e_{j}^{T} \\
-\frac{F_{j}^{+}+F_{j}^{-}}{2} e_{j} e_{j}^{T} & e_{j} e_{j}^{T}
\end{array}\right]\left[\begin{array}{l}
y\left(k-\tau_{1}(k)\right) \\
g\left(k-\tau_{1}(k)\right)
\end{array}\right] \\
& \left.-\sum_{i=1}^{n} l_{i}\left[\begin{array}{l}
y\left(k-\tau_{2}(k)\right) \\
g\left(k-\tau_{2}(k)\right)
\end{array}\right]^{T}\left[\begin{array}{cc}
F_{j}^{-} F_{j}^{+} e_{j} e_{j}^{T} & -\frac{F_{j}^{+}+F_{j}^{-}}{2} e_{j} e_{j}^{T} \\
-\frac{F_{j}^{+}+F_{j}^{-}}{2} e_{j} e_{j}^{T} & e_{j} e_{j}^{T}
\end{array}\right]\left[\begin{array}{l}
y\left(k-\tau_{2}(k)\right) \\
g\left(k-\tau_{2}(k)\right)
\end{array}\right]\right\} \\
& =E\left\{\alpha^{T}(k)\left(\Pi+\Xi+\Xi^{T}+\tau_{1}^{M} S Z_{1}^{-1} S^{T}+\tau_{2}^{M} T Z_{2}^{-1} T^{T}\right) \alpha(k)-\left[\begin{array}{c}
y(k) \\
g(k)
\end{array}\right]^{T}\left[\begin{array}{cc}
F_{1} D & -F_{2} D \\
-F_{2} D & D
\end{array}\right]\left[\begin{array}{l}
y(k) \\
g(k)
\end{array}\right]\right. \\
& \left.-\left[\begin{array}{c}
y\left(k-\tau_{1}(k)\right) \\
g\left(k-\tau_{1}(k)\right)
\end{array}\right]^{T}\left[\begin{array}{cc}
F_{1} H & -F_{2} H \\
-F_{2} H & H
\end{array}\right]\left[\begin{array}{l}
y\left(k-\tau_{1}(k)\right) \\
g\left(k-\tau_{1}(k)\right)
\end{array}\right]-\left[\begin{array}{l}
y\left(k-\tau_{2}(k)\right) \\
g\left(k-\tau_{2}(k)\right)
\end{array}\right]^{T}\left[\begin{array}{cc}
F_{1} L & -F_{2} L \\
-F_{2} L & L
\end{array}\right]\left[\begin{array}{l}
y\left(k-\tau_{2}(k)\right) \\
g\left(k-\tau_{2}(k)\right)
\end{array}\right]\right\} \\
& =E\left\{\alpha^{T}(k)\left(W_{1}+\Xi+\Xi^{T}+\tau_{1}^{M} S Z_{1}^{-1} S^{T}+\tau_{2}^{M} T Z_{2}^{-1} T^{T}\right) \alpha(k)\right\} \text {. }
\end{aligned}
$$


TABLE I

Computed MaXimum Delay Bound $\tau_{2}^{M}$ AND Feasible INTERVALS FOR DIFFERENT $\delta_{0}$

\begin{tabular}{c|c|c}
\hline & $\begin{array}{c}\text { Allowable values } \\
\text { of } \tau_{2}^{M}\end{array}$ & $\begin{array}{c}\text { Feasible variation } \\
\text { intervals }\end{array}$ \\
\hline $\begin{array}{c}\text { By Theorem1 } \\
\text { in [15] }\end{array}$ & not applicable & infeasible \\
\hline By Corollary 3 & not applicable & {$[4,24]$} \\
\hline $\begin{array}{c}\text { By Theorem 2, } \\
\delta_{0}=0.3\end{array}$ & 29 & {$[4,20], \quad[21,29]$} \\
\hline $\begin{array}{c}\text { By Theorem 2, } \\
\delta_{0}=0.6\end{array}$ & 33 & {$[4,20], \quad[21,33]$} \\
\hline $\begin{array}{c}\text { By Theorem 2, } \\
\delta_{0}=0.9\end{array}$ & 55 & {$[4,20], \quad[21,55]$} \\
\hline
\end{tabular}

\section{ILLUSTRATIVE EXAMPLE}

Consider the delayed discrete-time RNN system with the following parameters:

$$
\begin{aligned}
& C=\left[\begin{array}{cc}
0.8 & 0 \\
0 & 0.9
\end{array}\right] \quad A=\left[\begin{array}{cc}
-0.1 & 0 \\
-0.2 & -0.1
\end{array}\right] \\
& B=\left[\begin{array}{cc}
-0.5 & 0 \\
-0.2 & -0.4
\end{array}\right] \quad J=[0.3,0.2]^{T} .
\end{aligned}
$$

Take the activation functions as follows:

$$
f_{1}(s)=-0.05 \sin s-0.05 \quad f_{2}(s)=-0.05 \sin s-0.05 \text {. }
$$

Now assuming that the lower bound of $\tau(k)$ is 4 , according to Corollary 1, we obtain that the maximum allowable delay bound is 24 using Matlab LMI toolbox. However, there is no feasible solutions for this system using [15, Th. 1] when $\tau^{m}=4$ and $\tau^{M}=24$. This result demonstrates that our theorem is less conservative than [15, Th. 1]. Also, if the variation probability of $\tau(k)$ is known, the corresponding upper bound for the time delay can be solved by using Theorem 1 . Here, we assume $\tau_{1}^{m}=$ $4, \tau_{1}^{M}=20$, and $\tau_{2}^{m}=21$ and we intend to find the upper bound $\tau_{2}^{M}$ so that the system is MSS. The maximum allowable values of $\tau_{2}^{M}$ are given in Table I when the values of $\delta_{0}$ are $0.3,0.6$, and 0.9 , respectively. The feasible solutions for $\delta_{0}=0.9$ are given as follows:

$$
\begin{aligned}
P & =\left[\begin{array}{cc}
2160.3 & -0.9 \\
-0.9 & 2.4
\end{array}\right] \\
Q_{1} & =\left[\begin{array}{cc}
6.4021 & -0.0038 \\
-0.0038 & 0.0001
\end{array}\right] \\
Q_{2} & =\left[\begin{array}{cc}
3.7375 & -0.0004 \\
-0.0004 & 0.0026
\end{array}\right] \\
Z_{1} & =\left[\begin{array}{cc}
215.7341 & -0.1087 \\
-0.1087 & 1.1852
\end{array}\right] \\
Z_{2} & =\left[\begin{array}{cc}
96.9527 & -0.1465 \\
-0.1465 & 0.1807
\end{array}\right] \\
D & =\left[\begin{array}{cc}
2431.3 & 0 \\
0 & 7.4
\end{array}\right] \\
H & =\left[\begin{array}{cc}
3449.8 & 0 \\
0 & 8.7
\end{array}\right] \\
L & =\left[\begin{array}{cc}
642.8996 & 0 \\
0 & 1.2411
\end{array}\right] .
\end{aligned}
$$

TABLE II

FEASIBLE INTERVALS FOR DIFFERENT $\delta_{0}$

\begin{tabular}{l|cc|cc}
\hline & \multicolumn{2}{|c|}{$\tau_{2}^{m}=\tau_{1}^{M}+1$} & \multicolumn{2}{c}{$\tau_{2}^{m}=\tau_{1}^{M}+5$} \\
\hline $\boldsymbol{\delta}_{0}=0.3$ & {$[4,20]$,} & {$[21,29]$} & {$[4,20]$,} & {$[25,32]$} \\
\hline $\boldsymbol{\delta}_{0}=0.6$ & {$[4,20]$,} & {$[21,33]$} & {$[4,20]$,} & {$[25,35]$} \\
\hline $\boldsymbol{\delta}_{0}=0.9$ & {$[4,20]$,} & {$[21,55]$} & {$[4,20]$,} & {$[25,56]$}
\end{tabular}

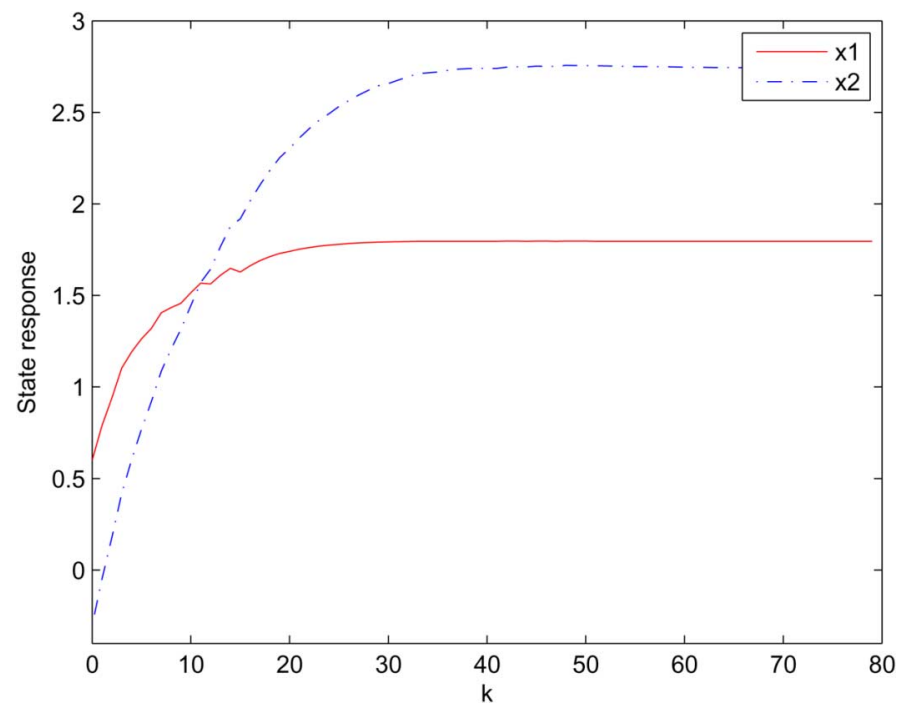

Fig. 1. Simulation of the system in (21) for $\delta_{0}=0.6$ and $\tau_{2}^{m}=\tau_{1}^{M}+1$.

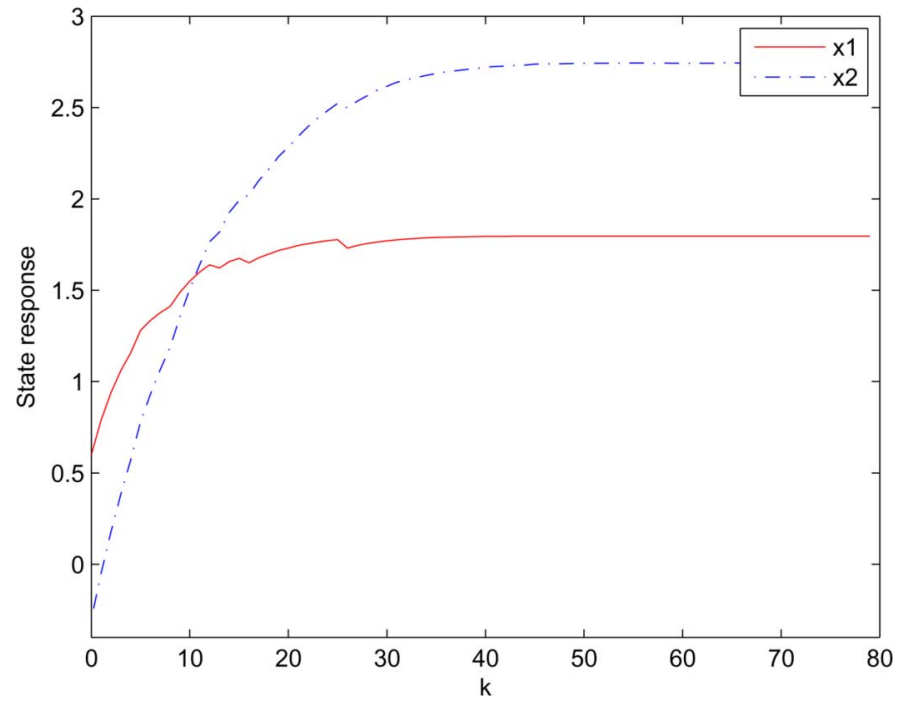

Fig. 2. Simulation of the system in (21) for $\delta_{0}=0.9$ and $\tau_{2}^{m}=\tau_{1}^{M}+5$.

It is illustrated in Table I that, when the variation probability of the delay is known, the maximum allowable delay bound may be increased. It is worth mentioning that our theorem is not limited to the special case when $\tau_{2}^{m}=\tau_{1}^{M}+1$. It allows a possible gap between the two intervals, which means $\tau_{2}^{m}>\tau_{1}^{M}+1$. To show this, we assume $\tau_{2}^{m}=\tau_{1}^{M}+5$, and the feasible intervals are given in Table II.

Fig. 1 is the simulation figure of the state response in (21) for $\delta_{0}=0.6$ when $\tau_{2}^{m}=\tau_{1}^{M}+1$. The initial condition of the system is $x(k)=[0.6,-0.3]^{T}$, where $-\tau \leq k \leq 0$ and $\tau=33$. Fig. 2 is for $\delta_{0}=0.9$ when $\tau_{2}^{m}=\tau_{1}^{M}+5$. The initial condition of the system is $x(k)=[0.6,-0.3]^{T}$, where $-\tau \leq k \leq 0$ and 
$\tau=56$. It is shown that all the state variables converge to their equilibrium point.

\section{CONCLUSION}

By defining a new Lyapunov function and by exploiting the probability distribution of the time-varying delay, we have obtained new results for the stability analysis of discrete-time RNNs with delay. The merit of the proposed results in this paper is that it has taken into consideration the probability distribution of the time delay. We have drawn a comparison with the previous result and demonstrated that when the information of distribution probability of time delay is available, the maximum allowable bound of the delay can be extended compared to the case when only the range of variation of the delay is known. Another advantage of our result is that it can deal with the case when a gap exists between the delay intervals. One important future research direction is to generalize our stability conditions to the case when there are more than two delay intervals.

\section{REFERENCES}

[1] K. Babcock and R. Westervelt, "Dynamics of simple electronic neural networks," Physica D: Nonlinear Phenomena, vol. 28, no. 3, pp. 305-316, 1987.

[2] J. Cao and J. Wang, "Global exponential stability and periodicity of recurrent neural networks with time delays," IEEE Trans. Circuits Syst. II, Reg. Papers, vol. 52, no. 5, pp. 920-931, May 2005.

[3] J. Cao, K. Yuan, and H. Li, "Global asymptotical stability of recurrent neural networks with multiple discrete delays and distributed delays," IEEE Trans. Neural Netw., vol. 17, no. 6, pp. 1646-1651, Nov. 2006.

[4] M. Coates and R. Nowark, "Network tomography for internal delay estimation," in Proc. IEEE Int. Conf. Acoust. Speech Signal Process., 2001, pp. 3409-3412.

[5] M. Coates and R. Nowark, "Sequential Monte Carlo inference of internal delays in nonstationary data networks," IEEE Trans. Signal Process., vol. 50, no. 2, pp. 366-376, Feb. 2002.

[6] H. Gao and T. Chen, "New results on stability of discrete-time systems with time-varying state delay," IEEE Trans. Autom. Control, vol. 52, no. 2, pp. 328-334, Feb. 2007.

[7] H. Gao, J. Lam, C. Wang, and Y. Wang, "Delay-dependent outputfeedback stabilization of discrete-time systems with time-varying state delay," Inst. Electr. Eng. Proc. D-Control Theory Appl., vol. 151, no. 6, pp. 691-698, 2004

[8] Y. He, Q. G. Wang, M. Wu, and C. Lin, "Delay-dependent state estimation for delayed neural networks," IEEE Trans. Neural Netw., vol. 17, no. 4, pp. 1077-1080, Jul. 2006.

[9] Y. He, M. Wu, and J. H. She, "An improved global asymptotic stability criterion for delayed cellular neural networks," IEEE Trans. Neural Netw., vol. 17, no. 1, pp. 250-252, Jan. 2006.

[10] S. Hu and J. Wang, "Global robust stability of a class of discrete-time interval neural networks," IEEE Trans. Circuits Syst. I, Reg. Papers, vol. 53, no. 1, pp. 129-129, Jan. 2006.

[11] E. Knightly, "H-bind: a new approach to providing statistical performance guarantees to VBR traffic," in Proc. IEEE INFOCOM, San Francisco, CA, Mar. 1996, pp. 1091-1099.

[12] J. Liang, J. Gao, and D. Ho, "Discrete-time bidirectional associative memory neural networks with variable delays," Phys. Lett. A, vol. 335, no. 2-3, pp. 226-234, 2005.

[13] J. Liang, J. Gao, and J. Lam, "Convergence of discrete-time recurrent neural networks," Int. J. Bifurcat. Chaos, vol. 15, no. 2, pp. 581-595, 2005.

[14] X. Liu, R. Martin, M. Wu, and M. Tang, "Delay-dependent robust stabilization of discrete-time systems with time-varying delay," Inst. Electr. Eng. Proc.-Control Theory Appl., vol. 153, pp. 689-702, 2006.

[15] Y. Liu, Z. Wang, A. Serrano, and X. Liu, "Discrete-time recurrent neural networks with time-varying delays: Exponential stability analysis," Phys. Lett. A, vol. 362, pp. 480-488, 2007.

[16] C. Marcus, F. Waugh, and R. Westervelt, "Nonlinear dynamics and stability of analog neural networks," Physica D: Nonlinear Phenomena, vol. 51, pp. 234-247, 1991.
[17] Y. Pan, H. Marquez, and T. Chen, "Stability of remote control systems with unknown time varying delays by LMI techniques," Int. J. Control, vol. 79, pp. 752-763, 2006.

[18] F. Presti, N. Duffield, and J. H. Towsley, "Multicast-based inference of network-internal delay distributions," IEEE Trans. Netw., vol. 10, no. 6, pp. 761-775, Dec. 2002.

[19] Q. Song and J. Gao, "Periodic solutions and its exponential stability of reaction-diffusion recurrent neural networks with continuously distributed delays," Nonlinear Anal.: Real World Appl., vol. 7, no. 1, pp. 65-80, 2006.

[20] E. Tian, D. Yue, Y. Zhang, and F. Liu, "Delay-dependent robust stability of stochastic T-S fuzzy systems with fast time-varying delay," in Proc. 4th Int. Conf. Impulsive Hybrid Dyn. Syst., 2007, pp. $809-816$.

[21] Y. Tsang, M. Coates, and R. Nowark, "Network delay tomography," IEEE Trans. Signal Process., vol. 51, no. 8, pp. 2125-2136, Aug. 2003.

[22] L. Wang and Z. Xu, "Sufficient and necessary conditions for global exponential stability of discrete-time recurrent neural networks," IEEE Trans. Circuits Syst. I, Reg. Papers, vol. 53, no. 6, pp. 1373-1380, Jun. 2006.

[23] S. Wang, R. Nathuji, R. Battati, and W. Zhao, "Providing statistical delay guarantees in wireless networks," in Proc. 24th Int. Conf. Distrib. Comput. Syst., 2004, pp. 48-55.

[24] Z. Wang, K. Fraser, and X. Liu, "Stochastic stability of uncertain Hopfield neural networks with discrete and distributed delays," Phys. Lett. A, vol. 354, no. 4, pp. 288-297, 2006.

[25] Z. Wang and Y. Liu, "On global asymptotic stability of neural networks with discrete and distributed delays," Phys. Lett. A, vol. 345, no. 4-6, pp. 299-308, 2005.

[26] Z. Wang, Y. Liu, M. Li, and X. Liu, "Stability analysis for stochastic Cohen-Grossberg neural networks with mixed time delays," IEEE Trans. Neural Netw., vol. 17, no. 3, pp. 814-820, May 2006.

[27] Z. Wang, Y. Liu, and L. Yu, "Exponential stability of delayed recurrent neural networks with Markovian jumping parameters," Phys. Lett. A, vol. 356, no. 4-5, pp. 346-352, 2006.

[28] Z. Wang, H. Shu, Z. Fang, and X. Liu, "Robust stability for stochastic Hopfield neural networks with time delays," Nonlinear Anal.: Real World Appl., vol. 7, no. 5, pp. 1119-1128, 2006.

[29] W. Xiong and J. Cao, "Global exponential stability of discrete-time Cohen-Grossberg neural networks," Neurocomputing, vol. 64, pp. 433-446, 2005.

[30] S. Xu, J. Lam, and D. Ho, "Novel global asymptotic stability criteria for delayed cellular neural networks," IEEE Trans. Circuits Syst. II, Reg. Papers, vol. 52, no. 6, pp. 349-353, Jun. 2005.

[31] S. Xu, J. Lam, and D. Ho, "A new LMI condition for delaydependent asymptotic stability of delayed Hopfield neural networks," IEEE Trans. Circuits Syst. II, Reg. Papers, vol. 53, no. 3, pp. 230-234, Mar. 2006.

[32] Z. Yuan, D. Hu, and L. Huang, "Stability and bifurcation analysis on a discrete-time neural network," Comput. Appl. Math., vol. 177, no. 1, pp. 89-89, 2005.

[33] D. Yue, Y. Zhang, and E. Tian, "Improved global robust delay-dependent stability criteria for delayed cellular neural networks," Int. J. Comput. Math., vol. 85, no. 8, pp. 1265-1277, 2008.

[34] H. Zhao and L. Wang, "Stability and bifurcation for discrete-time Cohen-Grossberg neural network," Appl. Math. Comput., vol. 179, no. 2, pp. 787-798, 2006.

[35] L. Zou and Z. Zhou, "Periodic solutions for nonautonomous discretetime neural networks," Appl. Math. Lett., vol. 19, no. 2, pp. 174-185, 2006.

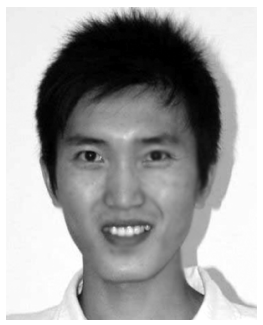

Yu Zhao was born in Heilongjiang, China, in 1985. $\mathrm{He}$ received the B.S. degree in automation from Harbin Institute of Technology, Harbin, China, in 2008. Currently, he is working towards the Ph.D. degree in electrical engineering at the University of Southern California, Los Angeles.

His current research interests include subspace identification methods, identification of delayed systems, process control, and process monitoring. 


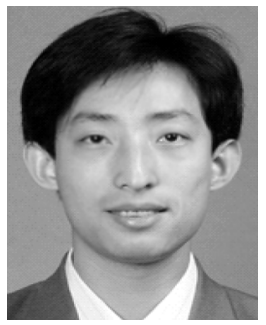

Huijun Gao was born in Heilongjiang, China, in 1976. He received the M.S. degree in electrical engineering from Shenyang University of Technology, Shenyang, China, in 2001 and the Ph.D. degree in control science and engineering from Harbin Institute of Technology, Harbin, China, in 2005.

From November 2003 to August 2004, he was a Research Associate at the Department of Mechanical Engineering, University of Hong Kong, Hong Kong. In November 2004, he joined Harbin Institute of Technology, where he is currently a Professor. From October 2005 to October 2007, he was a Postdoctoral Researcher at the Department of Electrical and Computer Engineering, University of Alberta, Edmonton, AB, Canada. His current research interests include network-based control, robust control, time-delay systems, and their industrial and aerospace applications.

Prof. Gao servers as Associate Editor for several international journals, including the IEEE TRANSACTIONS ON SYSTEMS, MAN AND CYBERNETICS-PART B: CYBERNETICS, the IEEE TRANSACTIONS ON InDUSTRIAL ElECTRONICS, Journal of Intelligent and Robotics Systems, International Journal of Systems Science. He was an outstanding reviewer for the IEEE TRANSACTIONS ON AUTOMATIC CONTROL and Automatica in 2008 and 2007, respectively, and an appreciated reviewer for the IEEE TRANSACTIONS ON Signal PROCESSING in 2006. He was the recipient of the University of Alberta Dorothy J. Killam Memorial Postdoctoral Fellow Prize in 2005, the National Outstanding Youth Science Fund in 2008, and the National Outstanding Doctoral Thesis Award in 2007. He was the corecipient of the National Natural Science Award of China in 2008. He is an Honorary Professor in the Department of Mechanical Engineering, University of Hong Kong.

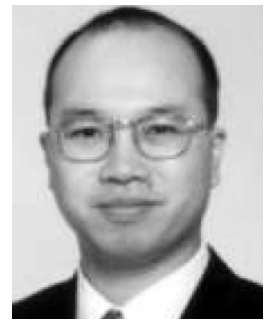

James Lam (S'86-M'87-SM'99) received the B.Sc. degree (with first class honors) in mechanical engineering from the University of Manchester, Manchester, U.K., and the M.Phil. and Ph.D. degrees in control engineering from the University of Cambridge, Cambridge, U.K

Currently, he is a Professor at the Department of Mechanical Engineering, University of Hong Kong, Hong Kong. His current research interests include reduced-order modeling, delay systems, descriptor sysrobust control, and filtering. tems, stochastic systems, multidimensional systems,

Dr. Lam received the Ashbury Scholarship, the A.H. Gibson Prize, and the $\mathrm{H}$. Wright Baker Prize for his academic performance. He is an Associate Editor of the Asian Journal of Control, International Journal of Systems Science, Journal of Sound and Vibration, International Journal of Applied Mathematics and Computer Science, the IEEE TRANSACtions on Signal Processing, Journal of the Franklin Institute, Dynamics of Continuous, Discrete and Impulsive Systems (Series B: Applications and Algorithms), and Automatica. $\mathrm{He}$ is also a member of the Editorial Board of the IET Control Theory and Applications, Open Electrical and Electronic Engineering Journal, Research Letters in Signal Processing, International Journal of Systems, Control and Communications, and Journal of Electrical and Computer Engineering. $\mathrm{He}$ was the Editor-in-Chief of the IEE Proceedings Control Theory and Applications. He is a Chartered Mathematician and a Chartered Scientist. He is a Fellow of the Institute of Mathematics and Its Applications, and the Institution of Engineering and Technology (IET). He is a Scholar and a Fellow of the Croucher Foundation.

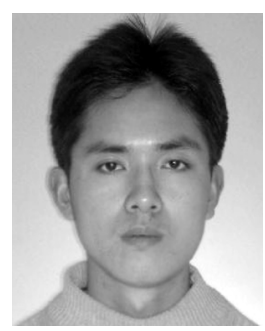

Ke Chen received the B.S. degree in mathematics and the M.S. degree in bioinformatics in 2005 from Nankai University, Tianjin, China. He is currently working towards the Ph.D. degree in electrical and computer engineering at the University of Alberta, Edmonton, AB, Canada.

His current research interests include application of mathematical models in biological sciences. 